\title{
Understanding Disorder in Rectangular Dielectric Microcavity CROWs (Project Report 0642603-Y2)
}

\author{
SHAYAN MOOKHERJEA \\ Department of Electrical and Computer Engineering, University of California, San Diego, MC 0407 La \\ Jolla CA 92093-0407 USA \\ Email:smookher@ucsd.edu
}

\section{Introduction and Goals}

This NSF-funded project [0642603] is a five-year (60 months) CAREER (Faculty Early Career Development Program) unified research and education development program, which focused on the physics and applications of optical waveguiding in the CROW (Coupled Resonator Optical Waveguide) structure. The CROW structure is suitable as the foundation of this project because it offers a very high four-wave mixing (FWM) nonlinearity based on the slowlight effects on each of the pump, signal and idler modes. The triple resonance effects can result in a large improvement of the nonlinear coefficient even with a modest improvement of the slowing factor. However, understanding the effects of disorder in CROWs is important, since it can limit the amount of slowing that can be achieved, and hence, the enhancement of slow-light enhanced nonlinearity.

\section{Activities}

Research this year (Y2, 02/08 - 01/09) focused on Task Area B: understanding the role that disorder plays in CROWs. Disorder can cause deviations from phase matching which are also more important in the slow-light regime [1]. Interesting physics phenomena such as localization of light can perhaps be more easily observed using the CROW structure as a testbed.

Previously, a tight-binding model of band-center propagation was formulated [2], [3], and theoretical studies of nonlinear propagation were developed on this basis [4], [5]. The third-order nonlinearity can also be used in other topics in nonlinear optics, such as bright and dark soliton formation [6], [7]. CROWs rely on lithography for optical confinement, and it was predicted that propagation in the regions of high dispersion and high nonlinearity would be sensitive to disorder [8]. Newer models were developed in this project [9] and were compared to earlier models and analyses [10].

We designed a CROW using one hundred coupled compact silicon microresonators as shown in Figure 1. The type of resonators we used for this study are known as rectangular dielectric microcavities based on a 2002 paper by Hammer [11]. Using a tunable laser near $1550 \mathrm{~nm}$, we measured band-edge 
light transmission properties. We were able to demonstrate Anderson localization of light due to small amounts of disorder by using a laser tuned to the band-edge of the CROW. The resonators are smaller than the pixel size of typical InGaAs cameras and it was difficult to image them clearly given the low optical transmission near the band edge. We developed a measurement technique for imaging modes which was sensitive to weak amounts of guided light, and also achieved high resolution. Our technique was based on confocal imaging and a scanning knife-edge method, followed by de-noising and digital differentiation. The experimentally measured localization length of 6 unit cells agreed closely with the theoretical estimate of 5.6 unit cells. The results were published in the journal Nature Photonics [12].

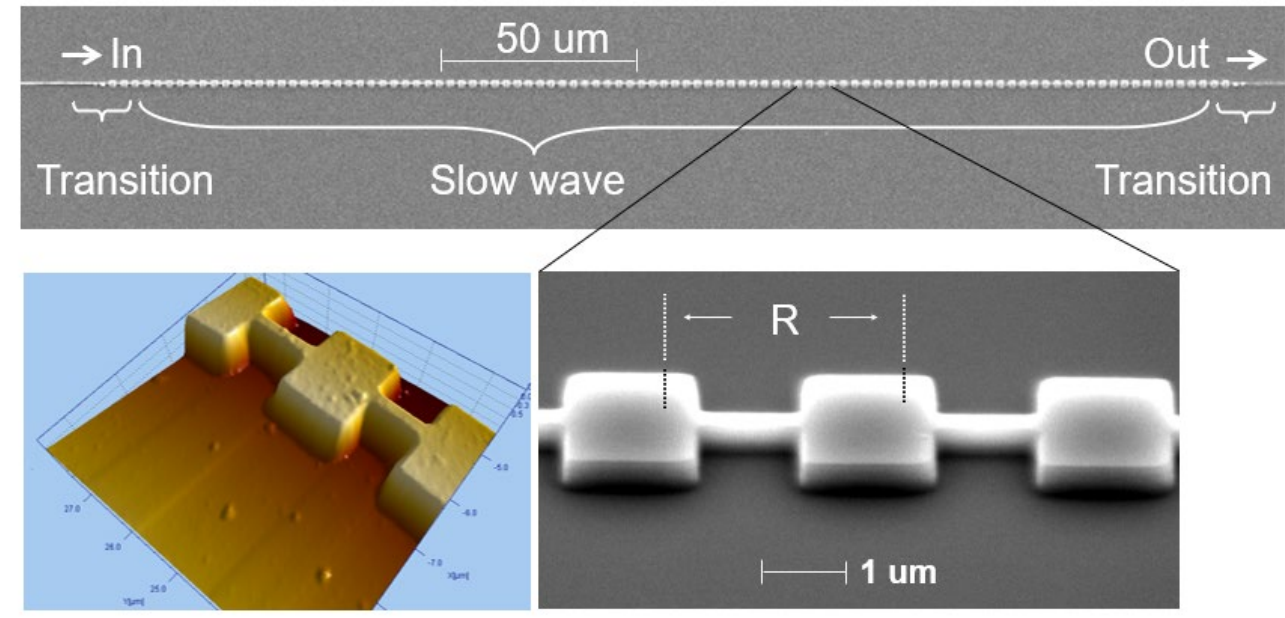

Figure 1 Scanning electron microscope and atomic-force microscope images of a CROW structure consisting of rectangular dielectric microcavities [9]. These structures were used to experimentally study band-edge localization of light.

\section{Progress and Impact}

Long CROWs: Long chains of coupled resonators were necessary to study localization because as the number of coherently coupled resonators in the chain grows, the separation between eigenvalues becomes smaller, and eventually becomes comparable to the disorder-induced perturbation (after taking into account the effects of exchange narrowing). We were able to achieve the longest-length CROW structures fabricated to date, by nearly a factor of 8 . Around the same time as this work, the silicon photonics group at IBM Research developed coupled microring CROWs also with up to one hundred unit cells. However, light propagation in those structures was noted to be highly non-uniform. Eye diagrams of modulated light could only be measured for much smaller lengths of CROWs. We initiated a collaboration with IBM to study and develop coupled-microring CROWs further, which eventually led to record-length CROWs with up to 235 unit cells. Led by the Notomi group at 
NTT in Japan, photonic crystal CROWs continued to progress over the next decade, and CROWs with more than 400 coupled photonic crystal cavities were realized and used for applications in quantum photonic information processing, such as correlated photon-pair generation and single-photon buffers [13].

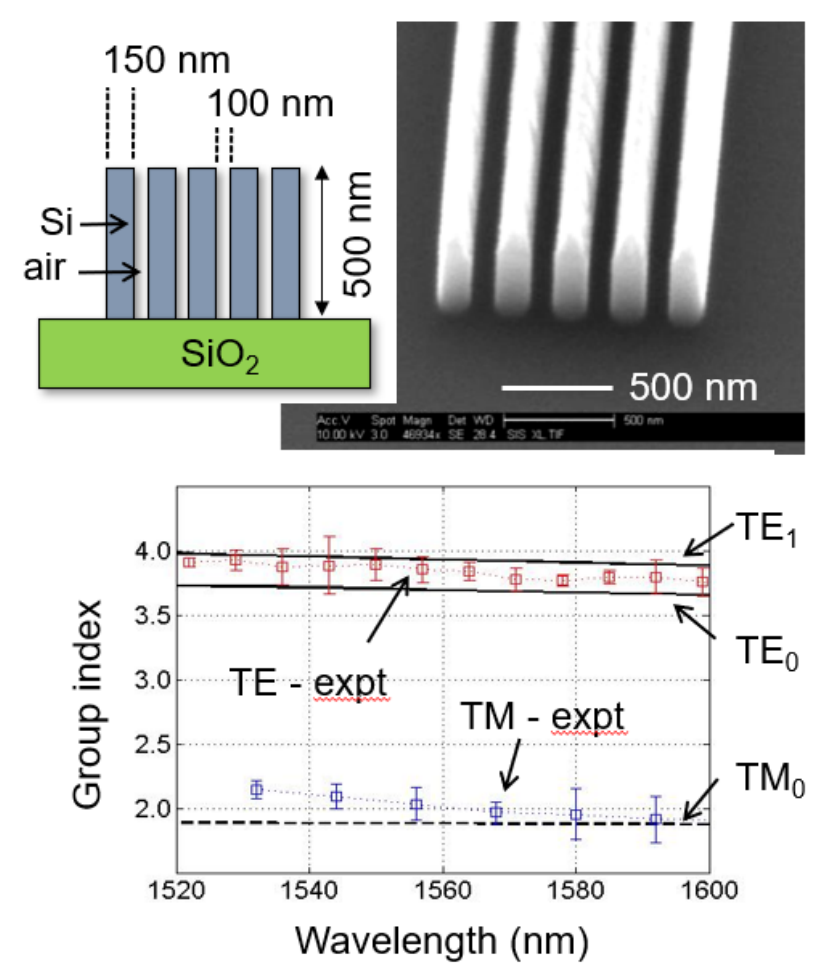

Figure 2 Giant birefringence in multi-slot silicon waveguides fabricated using electron-beam lithography and dry etching. The optical group index was measured by using such a waveguide in a Fabry-Perot resonator and measuring the spectrum using a swept-wavelength laser.

Slotted Waveguides: Another set of research activities this year was on nanophotonic "void" or "slotted" waveguides. Such structures comprise alternate layers of low index and high index material, with cross-sectional features smaller than the wavelength of light. One potential application is to engineer a high birefringence, i.e., a difference between the refractive indices of the TE and TM polarized modes, so that a compact polarization splitter can be realized. Prior to our work, single-slot waveguides have been proposed and experimentally demonstrated [14], and horizontally oriented multilayer structures had been studied through numerical simulations [15]. We designed, fabricated and demonstrated vertically striped multi-slotted waveguides (see Fig. 2) [16]. We experimentally measured the group index of fabricated multislotted waveguides in silicon in close agreement over a wide range of wavelengths with simulation results. To accomplish this, Fabry-Perot resonators were fabricated using these waveguide structures, and the transmission spectrum was measured over the $1520 \mathrm{~nm}-1600 \mathrm{~nm}$ range using 
a tunable laser. The Fourier transform of the transmission spectrum was analyzed to study the dispersion of the resonance peaks.

Other numerical simulations: We also studied a range of other optical structures through numerical simulations. An accurate knowledge of dispersion is also necessary for coupled-microring filters [17] or tunable dispersion compensation devices [18]. We experimentally studied the dispersion of directional couplers using a microring coupled to a waveguide [19]. To help in designing structures in silicon photonics, we developed an extension of coupled-mode theory which is applicable to directional couplers in high index contrast materials, in coupled-waveguide structures [20] and coupled-resonator structures [21]. Both the linear and the nonlinear regime are of importance. When the optical power is increased, silicon microring resonators with directional couplers can exhibit interesting behavior, such as bistability and nonlinear on-off switching [22].

Control over CROW waveguide properties: It has long been sought to dynamically tune the waveguiding properties of the CROW structure. Our studies focused on achieving electro-optic tunability for most of our studies. This was not feasible in the first batch of devices which we designed and fabricated, since we lacked the capability to realize p-n junctions part of the silicon photonics processing. However, through external collaborations, such structures were eventually fabricated. Using electro-optic control fields was practically convenient since it was easy to generate the switching waveforms in the laboratory [23]. Integrated electro-optic modulators could be another way of achieving phase shifters with more than $100 \mathrm{GHz}$ of bandwidth, as was demonstrated later [24]. If tuning speed is not a concern, the thermo-optic effect, which we used for tunable filters, is also another option as a control knob [25].

As research from NTT showed in the case of coupled photonic-crystal defect resonators, CROWs can be useful for generating correlated photon pairs [26]. In the context of coupled microring CROWs, we developed a model for spontaneous four-wave mixing (SFWM) using the Collett-Gardiner approach [27]. This method formulates the time-domain equations of motion in the Heisenberg picture. We solved the equations in the case of a single resonator as well as for multiple resonators, showing the potential advantages of the latter.

A collaboration with IBM's silicon photonics group (Dr. W. M. J. Green) provided insights into silicon photonics fabrication and also supported technical collaboration on fabricating the CROW structures [10], [28], [29].

We collaborated with Dr. Ivan B. Divliansky (Research Scientist, CREOL, Florida) for the electron-beam lithography of waveguide structures in silicon photonics [30]. We were able to write waveguides up to $5.9 \mathrm{~mm}$ length (40 e- 
beam write fields without stitching error) and achieve propagation loss less than $2.8 \mathrm{~dB} / \mathrm{cm}$.

CROWs were successfully fabricated and measured, and a good agreement was achieved between experiments and theory [29], [31], [32]. Infrared imaging is a useful experimental tool to study light propagation through the length of these structures [30], [33].

Impact on Human Resources: Three graduate students in the PI's group worked on this topic as part of their education and training. One of the students was supported by an NSF Graduate Fellowship. Two undergraduate students contributed to research projects.

\section{Subsequent Extensions}

Based on the outcomes of this CAREER project, a wide range of subsequent projects were initiated on focused research topics, including nonlinear mixers, harnessing disorder for functionality, and quantum photonics.

The CROW structure can have other applications including generation of quantum light [27], and developing amplifiers and lasers with novel properties [34]. The resonator-enhanced nonlinearity benefits the generation of entangled photon-pair and heralded single-photon generation using silicon photonics [35]. Our initial measurements of spontaneous four-wave mixing (SFWM) were on correlated photon-pair generation and heralded single-photon generation. An experimental measurement of entanglement was performed later, using a twophoton interferometer constructed to verify time-energy entanglement [35].

Measurements performed during this year showed the utility of being able to measure group index and dispersion rapidly. Subsequently, we developed novel measurement techniques for measuring these quantities, based on a single-scan, Jones matrix-based, interferometric spectral measurement instrument (Luna Tech. Optical Vector Analyzer OVA5000, generously loaned by Luna Technologies, VA. The commercial instrument was used in an innovative way in our measurements [36]. A jointly-authored publication resulted from this collaboration [10].

Studies performed in this project clearly showed that CROWs are highly sensitive to disorder. We studied methods for precisely tuning resonators without heaters, which cause crosstalk between adjacent resonators, especially due to the oxide layer beneath the silicon device layer, which acts as a thermal barrier to the substrate. One non-thermal tuning method which we developed is based on field-induced local oxidation of $\mathrm{Si}$ to $\mathrm{SiO}_{2}$ via a chemical reaction near an electrically-biased conducting atomic-force microscope tip [37]. Scanning probe lithography has previously been used to modify the resonance frequency of a GaAs photonic crystal cavity. However, this method is time consuming 
and can take hours to tune a single microring (using off-the-shelf instrumentation). Thus, a single silicon microresonator could be monitored and controlled more easily [38] than a long CROW device.

\section{Open-Access Reporting Initiative}

PRAISE: This open-access document is provided in support of our PRAISE

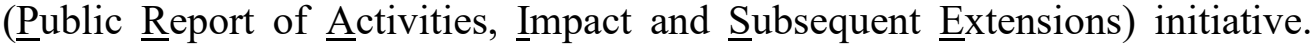
What is it? An open-access document shared with the public which describes the research outcomes of publicly-funded projects. For us, these projects are typically funded by the NSF (National Science Foundation).

\section{References}

[1] S. Hughes, L. Ramunno, J. F. Young, and J. E. Sipe, "Extrinsic Optical Scattering Loss in Photonic Crystal Waveguides: Role of Fabrication Disorder and Photon Group Velocity," Phys. Rev. Lett., vol. 94, no. 3, p. 033903, Jan. 2005, doi: 10.1103/PhysRevLett.94.033903.

[2] S. Mookherjea and A. Yariv, "Optical pulse propagation in the tightbinding approximation," Opt. Express, vol. 9, no. 2, p. 91, Jul. 2001, doi: 10.1364/OE.9.000091.

[3] S. Mookherjea and A. Yariv, "Pulse propagation in a coupled resonator optical waveguide to all orders of dispersion," Phys. Rev. E, vol. 65, no. 5, p. 056601, Apr. 2002, doi: 10.1103/PhysRevE.65.056601.

[4] S. Mookherjea and A. Yariv, "Kerr-stabilized super-resonant modes in coupled-resonator optical waveguides," Phys. Rev. E, vol. 66, no. 4, p. 046610, Oct. 2002, doi: 10.1103/PhysRevE.66.046610.

[5] S. Mookherjea and A. Yariv, "Optical pulse propagation and holographic storage in a coupled-resonator optical waveguide," Phys. Rev. E, vol. 64, no. 6, p. 066602, Nov. 2001, doi: 10.1103/PhysRevE.64.066602.

[6] B. Crosignani, A. Yariv, and S. Mookherjea, "Nonparaxial spatial solitons and propagation-invariant pattern solutions in optical Kerr media," Opt. Lett., vol. 29, no. 11, p. 1254, Jun. 2004, doi: 10.1364/OL.29.001254.

[7] A. Ciattoni, B. Crosignani, S. Mookherjea, and A. Yariv, "Nonparaxial dark solitons in optical Kerr media," Opt. Lett., vol. 30, no. 5, p. 516, Mar. 2005, doi: 10.1364/OL.30.000516.

[8] S. Mookherjea, D. S. Cohen, and A. Yariv, "Nonlinear dispersion in a coupled-resonator optical waveguide," Opt. Lett., vol. 27, no. 11, p. 933, Jun. 2002, doi: 10.1364/OL.27.000933.

[9] S. Mookherjea and A. Oh, "Effect of disorder on slow light velocity in optical slow-wave structures," Opt. Lett., vol. 32, no. 3, p. 289, Feb. 2007, doi: 10.1364/OL.32.000289. 
[10] M. L. Cooper et al., "Waveguide dispersion effects in silicon-oninsulator coupled-resonator optical waveguides," Opt. Lett., vol. 35, no. 18, p. 3030, Sep. 2010, doi: 10.1364/OL.35.003030.

[11] M. Hammer, "Resonant coupling of dielectric optical waveguides via rectangular microcavities: the coupled guided mode perspective," Optics Communications, vol. 214, no. 1-6, pp. 155-170, Dec. 2002, doi: 10.1016/S0030-4018(02)02171-5.

[12] S. Mookherjea, J. S. Park, S.-H. Yang, and P. R. Bandaru, "Localization in silicon nanophotonic slow-light waveguides," Nature Photon, vol. 2, no. 2, pp. 90-93, Feb. 2008, doi: 10.1038/nphoton.2007.278.

[13] N. Matsuda, H. Takesue, W. J. Munro, E. Kuramochi, and M. Notomi, "Photonic Quantum Information Devices Using Coupled-resonator Optical Waveguides," NTT Review, vol. 12, no. 9, p. 1, 2014.

[14] Q. Xu, V. R. Almeida, R. R. Panepucci, and M. Lipson, "Experimental demonstration of guiding and confining light in nanometer-size lowrefractive-index material," Opt. Lett., vol. 29, no. 14, p. 1626, Jul. 2004, doi: 10.1364/OL.29.001626.

[15] N.-N. Feng, J. Michel, and L. C. Kimerling, "Optical Field Concentration in Low-Index Waveguides," IEEE J. Quantum Electron., vol. 42, no. 9, pp. 883-888, Sep. 2006, doi: 10.1109/JQE.2006.880061.

[16] S.-H. Yang, M. L. Cooper, P. R. Bandaru, and S. Mookherjea, "Giant birefringence in multi-slotted silicon nanophotonic waveguides," Opt. Express, vol. 16, no. 11, p. 8306, May 2008, doi: 10.1364/OE.16.008306.

[17] J. R. Ong, R. Kumar, and S. Mookherjea, "Silicon microring-based wavelength converter with integrated pump and signal suppression," Opt. Lett., vol. 39, no. 15, p. 4439, Aug. 2014, doi: 10.1364/OL.39.004439.

[18] S. Mookherjea, "Using gain to tune the dispersion relation of coupledresonator optical waveguides," IEEE Photon. Technol. Lett., vol. 18, no. 5, pp. 715-717, Mar. 2006, doi: 10.1109/LPT.2006.871144.

[19] R. Aguinaldo, Yiran Shen, and S. Mookherjea, "Large Dispersion of Silicon Directional Couplers Obtained via Wideband Microring Parametric Characterization," IEEE Photon. Technol. Lett., vol. 24, no. 14, pp. 1242-1244, Jul. 2012, doi: 10.1109/LPT.2012.2198639.

[20] M. L. Cooper and S. Mookherjea, "Numerically-assisted coupledmode theory for silicon waveguide couplers and arrayed waveguides," Opt. Express, vol. 17, no. 3, p. 1583, Feb. 2009, doi: 10.1364/OE.17.001583.

[21] S. Mookherjea, "Spectral characteristics of coupled resonators," $J$. Opt. Soc. Am. B, vol. 23, no. 6, p. 1137, Jun. 2006, doi: 10.1364/JOSAB.23.001137. 
[22] S. Mookherjea and M. A. Schneider, "The nonlinear microring adddrop filter," Opt. Express, vol. 16, no. 19, p. 15130, Sep. 2008, doi: 10.1364/OE.16.015130.

[23] S. Mookherjea, J. R. Ong, X. Luo, and L. Guo-Qiang, "Electronic control of optical Anderson localization modes," Nature Nanotech, vol. 9, no. 5, pp. 365-371, May 2014, doi: 10.1038/nnano.2014.53.

[24] X. Wang, P. O. Weigel, J. Zhao, M. Ruesing, and S. Mookherjea, "Achieving beyond-100-GHz large-signal modulation bandwidth in hybrid silicon photonics Mach Zehnder modulators using thin film lithium niobate," APL Photonics, vol. 4, no. 9, p. 096101, Sep. 2019, doi: $10.1063 / 1.5115243$.

[25] R. Aguinaldo et al., "Wideband silicon-photonic thermo-optic switch in a wavelength-division multiplexed ring network," Opt. Express, vol. 22, no. 7, p. 8205, Apr. 2014, doi: 10.1364/OE.22.008205.

[26] N. Matsuda, H. Takesue, K. Shimizu, Y. Tokura, E. Kuramochi, and M. Notomi, "Slow light enhanced correlated photon pair generation in photonic-crystal coupled-resonator optical waveguides," Optics Express, vol. 21, no. 7, pp. 8596-8604, 2013.

[27] J. R. Ong and S. Mookherjea, "Quantum light generation on a silicon chip using waveguides and resonators," Opt. Express, vol. 21, no. 4, p. 5171, Feb. 2013, doi: 10.1364/OE.21.005171.

[28] J. R. Ong et al., "Low-power continuous-wave four-wave mixing in silicon coupled-resonator optical waveguides," Opt. Lett., vol. 36, no. 15, pp. 2964-2966, 2011.

[29] M. L. Cooper et al., "235-ring Coupled-Resonator Optical Waveguides," in Conference on Lasers and Electro-Optics 2010, San Jose, California, 2010, p. CTuHH3. doi: 10.1364/CLEO.2010.CTuHH3.

[30] M. L. Cooper, G. Gupta, J. S. Park, M. A. Schneider, I. B. Divliansky, and S. Mookherjea, "Quantitative infrared imaging of silicon-on-insulator microring resonators," Opt. Lett., vol. 35, no. 5, p. 784, Mar. 2010, doi: 10.1364/OL.35.000784.

[31] S. Mookherjea and M. A. Schneider, "Avoiding bandwidth collapse in long chains of coupled optical microresonators," Opt. Lett., vol. 36, no. 23, p. 4557, Dec. 2011, doi: 10.1364/OL.36.004557.

[32] M. L. Cooper and S. Mookherjea, "Modeling of Multiband Transmission in Long Silicon Coupled-Resonator Optical Waveguides," IEEE Photon. Technol. Lett., vol. 23, no. 13, pp. 872-874, Jul. 2011, doi: 10.1109/LPT.2011.2141657.

[33] S. Mookherjea and H. R. Grant, "High dynamic range microscope infrared imaging of silicon nanophotonic devices," Opt. Lett., vol. 37, no. 22, p. 4705, Nov. 2012, doi: 10.1364/OL.37.004705. 
[34] S. Mookherjea, "Semiconductor coupled-resonator optical waveguide laser," Appl. Phys. Lett., vol. 84, no. 17, pp. 3265-3267, Apr. 2004, doi: 10.1063/1.1719278.

[35] R. Kumar, M. Savanier, J. R. Ong, and S. Mookherjea, "Entanglement measurement of a coupled silicon microring photon pair source," Opt. Express, vol. 23, no. 15, p. 19318, Jul. 2015, doi: 10.1364/OE.23.019318.

[36] M. L. Cooper, M. A. Schneider, G. Gupta, and S. Mookherjea, "FilterLess Amplification for Rapid Swept-Wavelength Interferometric Measurement of Silicon Waveguide Group Delay Statistics," IEEE Photon. Technol. Lett., vol. 23, no. 12, pp. 783-785, Jun. 2011, doi: 10.1109/LPT.2011.2134842.

[37] Y. Shen, I. B. Divliansky, D. N. Basov, and S. Mookherjea, "Perfect set-and-forget alignment of silicon photonic resonators and interferometers," in Optical Fiber Communication Conference/National Fiber Optic Engineers Conference 2011, Los Angeles, California, 2011, p. PDPC3. doi: 10.1364/OFC.2011.PDPC3.

[38] M. Savanier, R. Kumar, and S. Mookherjea, "Optimizing photon-pair generation electronically using a $p-i-n$ diode incorporated in a silicon microring resonator," Appl. Phys. Lett., vol. 107, no. 13, p. 131101, Sep. 2015, doi: 10.1063/1.4932047. 